\title{
LONG-TERM STABILIZATION OF VEIN GRAFT WALL ARCHITECTURE AND PROLONGED RESISTANCE TO EXPERIMENTAL ATHEROSCLEROSIS AFTER E2F DECOY OLIGONUCLEOTIDE GENE THERAPY
}

Afshin Ehsan, MD

Michael J. Mann, MD

Giorgio Dell' Acqua, $\mathrm{PhD}$

Victor J. Dzau, MD
Objective: We tested the hypothesis that a single intraoperative transfection of rabbit vein grafts with a decoy oligonucleotide that blocks cell-cycle gene transactivation by the transcription factor E2F induces long-term stable adaptation that involves medial hypertrophy and a resistance to neointimal hyperplasia and atherosclerosis.

Methods: Jugular vein to carotid artery interposition vein grafts in hypercholesterolemic rabbits were treated, using pressure-mediated delivery, with either E2F decoy oligonucleotide, scrambled oligonucleotide, or vehicle alone. E2F decoy inhibition of cell-cycle gene expression was determined by measuring proliferating cell nuclear antigen upregulation and bromodeoxyuridine incorporation in vascular smooth muscle cells. Neointimal hyperplasia and atherosclerosis were compared between groups at 6 months after operation. Wall stress was derived from the ratio of luminal radius to wall thickness. Normal rabbits exposed to 6 weeks of dietinduced hypercholesterolemia starting 6 months after operation were analyzed in the same manner.

Results: The E2F decoy oligonucleotide, but not scrambled oligonucleotide or vehicle alone, inhibited proliferating cell nuclear antigen expression and smooth muscle cell proliferation. Furthermore, this manipulation of cellcycle gene expression yielded an inhibition of neointimal hyperplasia and atherosclerotic plaque formation throughout the 6 months of cholesterol feeding. In normocholesterolemic rabbits, vehicle-treated and scrambled oligonucleotide-treated vein grafts remain susceptible to diet-induced atherosclerosis as well, whereas resistance to this disease induction remained stable in genetically engineered grafts.

Conclusion: A single intraoperative pressure-mediated delivery of E2F decoy effectively provides vein grafts with long-term resistance to neointimal hyperplasia and atherosclerosis. These findings suggest that long-term reduction in human vein graft failure rates may be feasible with this ex vivo gene therapy approach. (J Thorac Cardiovasc Surg 2001;121:714-22)
A lthough autologous vein remains the most commonly used conduit for coronary and peripheral bypass grafting, vein graft occlusion rates of $30 \%$ to

From the Division of Cardiovascular Medicine, Brigham and Women's Hospital/Harvard Medical School, Boston, Mass.

This work was supported by National Institutes of Health grants HL61661, HL58516, HL59316, HL35610; the Charles and Ellen Collins Fund; and the Edna Mandel Fund. Dr Ehsan is the recipient of the American Heart Association post-doctoral fellowship grant 9820007T. Dr Mann is supported by the William Randolph Hearst Endowment for Young Investigators. Dr Dzau is the recipient of a National Heart, Lung, and Blood Institute MERIT Award.

Copyright (C) 2001 by The American Association for Thoracic Surgery $0022-5223 / 2001 \$ 35.00+0 \quad \mathbf{1 2 / 1 / 1 1 1 2 0 4}$

doi:10.1067/mtc.2001.111204
$50 \%$ persist, primarily because of neointimal hyperplasia and subsequent accelerated atherosclerosis. ${ }^{1-3}$ These vein graft failures result in significant morbidity and mortality and represent a major limitation in the longterm treatment of ischemic arterial disease.

Whereas inhibition of human neointimal hyperplasia has eluded traditional pharmacologic approaches, ${ }^{4}$ a growing understanding of the molecular biology of vascular cell activation and proliferation has allowed the design of interventions at the level of gene expression that have successfully altered the course of experimental vein graft disease. ${ }^{5,6} \mathrm{We}$ have previously reported that blockade of cell-cycle regulatory genes through intraoperative transfection of a combination of antisense oligodeoxynucleotides (ODNs) inhibited neointi- 


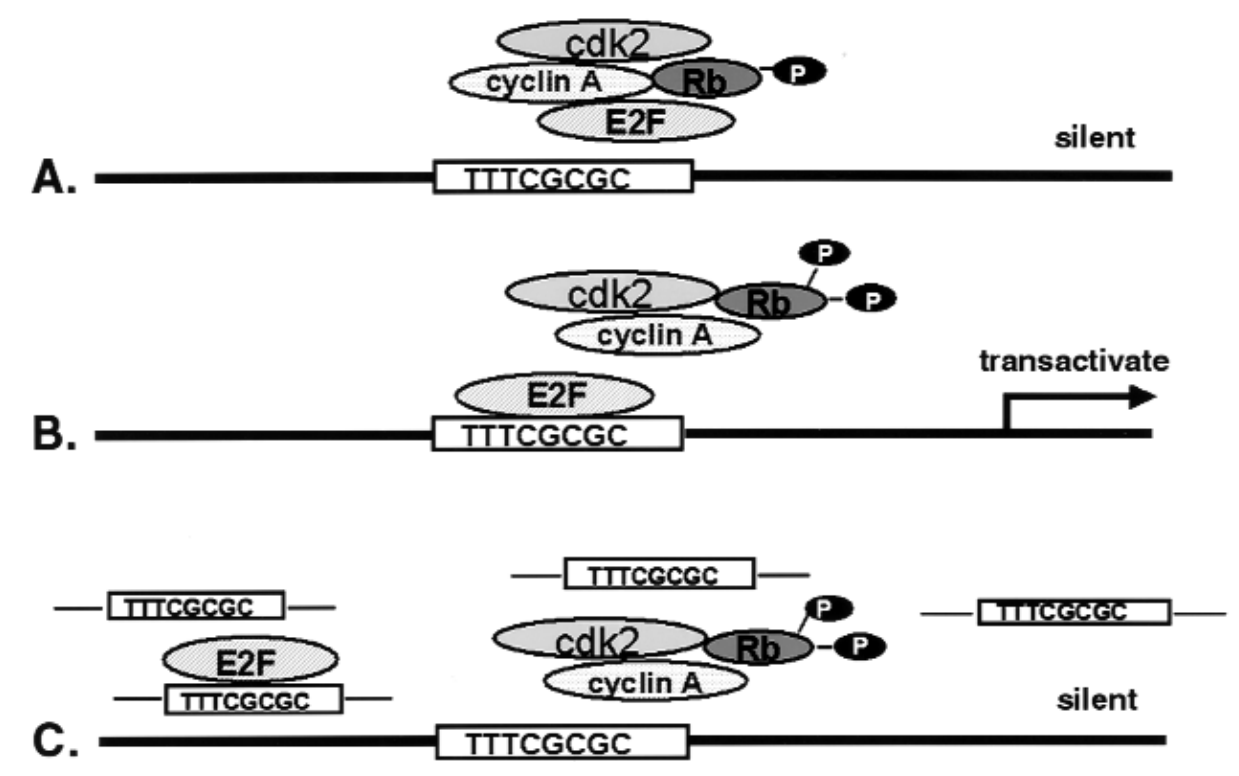

Fig 1. Principle of E2F decoy strategy (TTTCGCGC, consensus sequence for the E2F binding site). A, In a quiescent cell state the transcription factor $\mathrm{E} 2 \mathrm{~F}$ is complexed with retinoblastoma gene product $(R b)$, cyclin $\mathrm{A}$, and cyclin-dependent kinase-2 ( $c d K 2$ ). B, Hyperphosphorylation of retinoblastoma gene product releases free E2F, which binds to enhancer regions of cell-cycle regulatory genes, resulting in their transactivation. C, The E2F decoy double-stranded ODN binds to free E2F, preventing E2F-mediated transactivation of cell-cycle regulatory genes.

mal hyperplasia in rabbit vein grafts for up to 10 weeks after implantation. ${ }^{7}$ Adaptive wall thickening and reduction of wall stress was instead achieved through hypertrophy of the medial layer, and, together with a stabilization of endothelial function, this alteration in graft wall architecture prevented the development of accelerated diet-induced atherosclerosis.

The E2F-1 transcription factor is released from a regulatory complex involving the retinoblastoma gene product on the latter's hyperphosphorylation at a critical juncture in the late $G_{1}$ phase of the cell cycle. ${ }^{8}$ Free E2F has been associated with the upregulated expression of a dozen genes involved in subsequent DNA synthesis and cell-cycle progression (Fig 1). ${ }^{9-11}$ Our laboratory has previously documented that blockade of this transcription factor with a double-stranded decoy ODN bearing the consensus E2F-binding site inhibits the upregulation of multiple cell-cycle genes in vascular cells in a sequence-specific and coordinated fashion. ${ }^{12}$

In this study we tested the hypothesis that singleagent genetic treatment of vein grafts with E2F decoy ODNs not only redirects graft biology away from neointimal hyperplasia and toward medial hypertrophy as an adaptive response to arterial hemodynamics but also provides a stabilization of the graft wall that endows the vessel with long-term resistance to neointimal and atherosclerotic disease. This genetic manipula- tion was achieved ex vivo by using a nondistending pressure-mediated delivery system ${ }^{13}$ without the use of liposomes or viral vectors and may therefore represent a particularly safe approach toward the genetic engineering of human bypass grafts.

\section{Materials and methods}

Oligonucleotides. The double-stranded E2F decoy phosphorothioate ODN was custom synthesized (KeystoneBiosource, Menlo Park, Calif) with the sequence 5'-CTAGATTTCCCGCG-3', which contains the E2F consensus binding sequence found in the human $c$-myc promoter. The scrambled ODN used as a control in this study has the following sequence: 5'-TCCAGCTTCGTAGC-3'. ODN was dissolved at a concentration of $40 \mu \mathrm{mol} / \mathrm{L}$ in normal saline solution. Scrambled ODN labeled with fluorescein isothiocyanate at the $3^{\prime}$ end of one strand was also used for fluorescent microscopic analysis of ODN distribution after transfection.

Vein graft model and ex vivo transfection. New Zealand white rabbits $(3-3.5 \mathrm{~kg})$ were anesthetized with an intravenous mixture of ketamine $(2.5 \mathrm{mg} / \mathrm{mL})$ and xylazine $(0.09$ $\mathrm{mg} / \mathrm{mL}$ ). Using the no-touch technique, a $2.5-\mathrm{cm}$ segment of the right jugular vein was dissected through a midline vertical neck incision, and all branches were carefully ligated with 4-0 silk sutures and divided. Grafts were then cannulated distally and flushed gently with normal saline solution. The ends of the cannula and vein were then surrounded by an inelastic sheath, and ODN solution or vehicle alone was infused through the lumen of the vessel and into the surrounding 
space. The open end of the sheath was then clamped, and the fluid surrounding the vein was pressurized to $300 \mathrm{~mm} \mathrm{Hg}$. The graft was incubated under this nondistending pressure for 20 minutes, at which time the tissue was flushed again with normal saline solution to remove excess ODN solution. Animals were heparinized (200 U/kg), the ipsilateral common carotid artery was clamped distally and proximally, and the grafts were anastomosed into the divided artery with interrupted 7-0 polypropylene sutures. All grafts were treated with 1 to $2 \mathrm{~mL}$ of $2 \%$ lidocaine solution before wound closure. Our use of animals in this study was approved by the Harvard Medical Area Standing Committee on Animals, and all animal care complied with the "Guide for the Care and Use of Laboratory Animals," Institute of Laboratory Animal Resources, Commission on Life Sciences, National Research Council (National Academy Press, 1996).

Cholesterol feeding. One group of animals was started on a $1 \%$ cholesterol diet (Dyets, Inc, Bethlehem, Pa) 1 week before the operation and maintained on that diet until the time of death. A second group of animals was started on a $1 \%$ cholesterol diet 6 months after vein grafting and maintained on that diet for 6 weeks, at which time the animal was killed. Serum cholesterol levels in cholesterol-fed animals ranged from 800 to $2000 \mathrm{mg} / \mathrm{dL}$ during the study period (normocholesterolemia being 30-75 mg/dL).

Vessel morphometry. Vein grafts and ungrafted jugular veins were perfusion fixed at $100 \mathrm{~mm} \mathrm{Hg}$ and harvested at 6 weeks and 6 months, and paraffin-embedded sections were stained with hematoxylin-van Geison or with Verhoff-van Geison stain. Computerized planimetry (model 2200; South Micro Instruments, Atlanta, Ga) was used to measure the intimal and medial cross-sectional surface areas of each vessel spaced at $0.75-\mathrm{mm}$ intervals. Neointima was distinguished by its position relative to the internal elastic lamina and by its chaotic organization of cells compared with the circumferential arrangement of medial smooth muscle cells. Luminal radius and intimal and medial thickness were derived from these data, and averages for each vessel were calculated. The ratio of luminal radius to wall thickness is proportional to tangential wall stress by Laplace's law and was derived as an indication of tangential wall stress in these vessels.

Bromodeoxyuridine incorporation and immunohistochemistry. An index of cellular proliferation was obtained through the administration of bromodeoxyuridine (BrdU) at 18 hours (100 mg/kg subcutaneous and $30 \mathrm{mg} / \mathrm{kg}$ intravenous) and 12 hours (30 mg/kg intravenous) before harvest. On harvest, vein grafts are flushed with phosphate-buffered saline solution, pressure fixed at $100 \mathrm{~mm} \mathrm{Hg}$ with $10 \%$ neutral-buffered formalin for 10 minutes, and snap-frozen in OCT. Immunohistochemical staining with a BrdU-labeling kit (Zymed; San Francisco, Calif) was carried out on $5-\mu \mathrm{m}$ sections. Eight fields per section were counted at $400 \times$ magnification. The contralateral jugular vein was harvested and analyzed in the same manner as a control vein. Nuclei were counterstained with hematoxylin and counted to obtain a labeling index.

Western blot analysis. Whole graft explanted 4 days after operation was snap-frozen and ground into a powder form and lysed in an NP-40-based buffer (1\% NP-40, 0.5\% sodi- um deoxycholate, and $0.1 \%$ sodium dodecylsulfate) containing protease inhibitors $(10 \mu \mathrm{g} / \mathrm{mL}$ phenylmethylsulfonyl fluoride and $30 \mu \mathrm{g} / \mathrm{mL}$ aprotinin) and the phosphatase inhibitor sodium orthovanadate $(1 \mathrm{mmol} / \mathrm{L}$; Sigma Chemical Co, St Louis, Mo). The insoluble fraction was removed by centrifugation. Proteins were separated in a $10 \%$ sodium dodecyl sulfate-polyacrylamide gel and transferred to a nitrocellulose membrane (Hybond ECL; Amersham Life Sciences, Piscataway, NJ). The membrane was incubated with a polyclonal antibody used for proliferating cell nuclear antigen (PCNA; Santa Cruz Biotechnology Inc, Santa Cruz, Calif) for 1 hour and developed by using the ECL system (Amersham Life Sciences). Computerized image analysis was used to quantify the relative signal intensities of the bands using the software NIH Image 1.52 (Bethesda, Md), and the results were expressed in arbitrary units per microgram of protein.

Statistical analysis. All results are expressed as the mean \pm SD. One-way analysis of variance followed by the Bonferroni multiple comparison test was used to compare differences between groups.

\section{Results}

Inhibition of cell-cycle regulatory elements by E2F decoy ODN. E2F decoy or scrambled ODN was delivered to graft vascular smooth muscle cells (VSMCs) in a highly efficient manner by using nondistending pressure-mediated transfection, as previously described. ${ }^{13}$ Previous reports have documented a transfection efficiency of approximately $90 \%$ of vascular cells in vein grafts with this ex vivo approach, and this efficiency of transfection was confirmed in this study by using fluorescein isothiocyanate-labeled ODN (data not shown). Effective delivery of functional E2F decoy ODN was confirmed by means of Western blot analysis for PCNA in whole graft protein lysates 4 days after treatment and implantation. We determined that grafts treated with the E2F decoy ODN had a sequence-specific inhibition of PCNA upregulation when compared with vehicle or scrambled ODN-treated grafts (Fig 2, $A$ and $B$ ). Furthermore, grafts harvested 7 days after implantation, a time when VSMC proliferation is known to be at its peak, were assessed for BrdU incorporation in medial cells on 5-mm cross-sections. These findings once again confirmed that grafts treated with E2F decoy ODN had an inhibition of medial VSMC proliferation, as determined by a significantly lower BrdU-labeling index, when compared with vehicle and scrambled ODN-treated control grafts (Fig 2,C).

Long-term graft wall stabilization and inhibition of atherosclerosis. Treatment of grafts with E2F decoy ODN led to a significant reduction in neointimal thick- 
A.
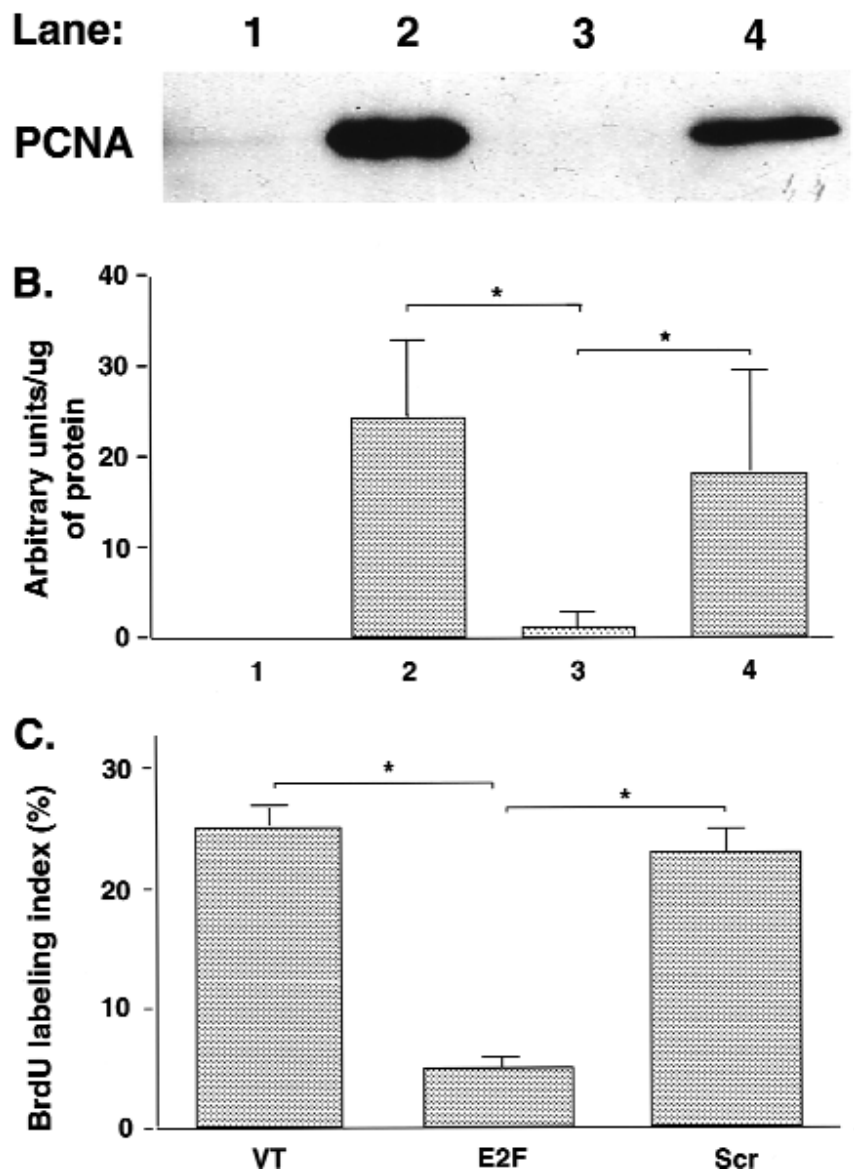

Fig 2. Effect of E2F decoy ODN on PCNA expression and BrdU-labeling index in vein grafts. A, Representative Western blot demonstrating PCNA protein expression of whole-tissue lysates in ungrafted jugular vein (lane 1) and in day 4 grafts treated with vehicle (lane 2), E2F decoy ODN (lane 3), or scrambled ODN (lane 4). B, Significant differences in expression were found between E2F decoy ODN-treated and vehicle-treated or scrambled ODNtreated grafts $\left(\mathrm{n}=6\right.$ for each group, $\left.{ }^{*} P<.017\right)$. Numbers on the $\mathrm{x}$ axis refer to lanes as defined in A. C, E2F decoy ODN treatment significantly inhibits BrdU incorporation when compared with vehicle-treated or scrambled ODNtreated groups ( $\mathrm{n}=3$ for each group, ${ }^{*} P<.017$ ). $V T$, Vehicle-treated graft; $E 2 F$, E2F decoy ODN-treated graft; $S c r$, scrambled ODN-treated graft.

ness at 6 weeks and 6 months $(11 \pm 12$ and $13 \pm 3 \mu \mathrm{m}$, respectively) compared with vehicle-treated $(122 \pm 12$ and $151 \pm 12 \mu \mathrm{m}$, respectively) or scrambled ODNtreated (131 \pm 9 and $150 \pm 10 \mu \mathrm{m}$, respectively) grafts. Instead, the E2F decoy ODN-treated grafts responded to the increase in hemodynamic stress of the arterial circulation through a thickening of the medial layer (Fig 3). The hemodynamic stability achieved by this medial thickening is reflected in the reduction of wall stress in E2F decoy ODN-treated grafts to a level similar to those seen in the carotid artery and in the control grafts that underwent significant neointimal hyperplasia (Fig 4).
At 6 months after grafting, extensive plaque was noted throughout the lengths of all vehicle-treated and scrambled ODN-treated grafts placed in cholesterolfed rabbits. By contrast, the E2F decoy ODN-treated grafts remained largely free of macroscopic plaque, as did the ipsilateral grafted and contralateral ungrafted carotid arteries. Histologic examination of atherosclerotic grafts in control animals revealed lesions rich in foam cells, many of which were covered by a fibrous cap of varying cellularity (Fig 5). These complex lesions were absent in E2F decoy ODN-treated grafts, in which only occasional foam cells were identified. Furthermore, the shift in vein graft adaptation seen at 


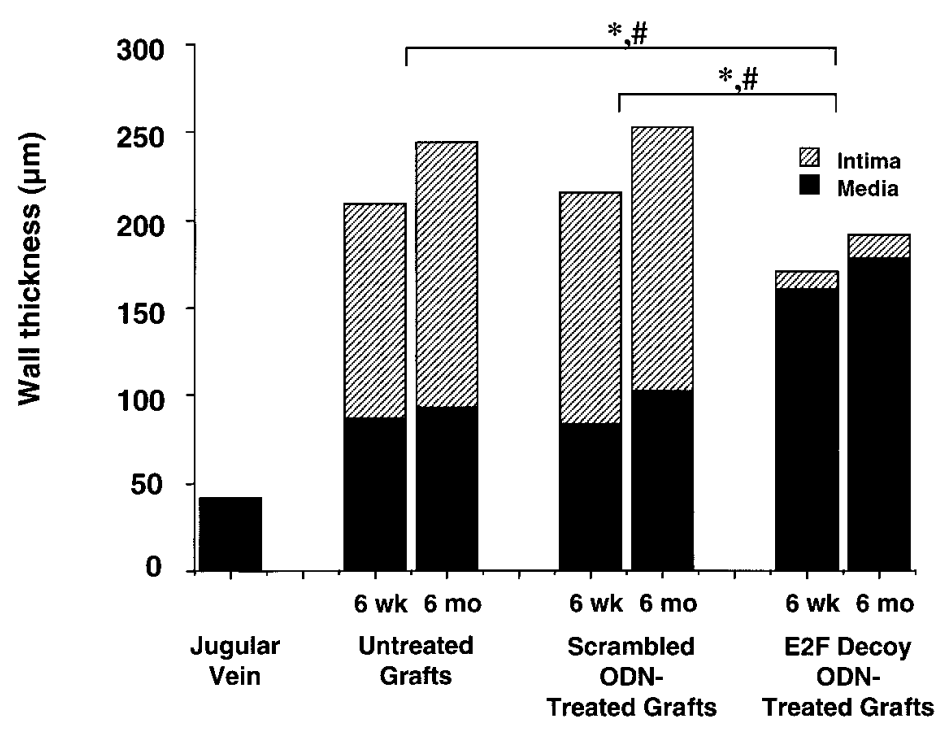

Fig 3. Neointimal, medial, and total wall thickness at 6 weeks and 6 months after placement of vehicle-treated, scrambled ODN-treated, and E2F decoy ODN-treated vein grafts into cholesterol-fed rabbits. Stable adaptive wall thickening is achieved in E2F decoy ODN-treated grafts through medial hypertrophy with a minimal degree of neointimal hyperplasia that does not increase over time $(\mathrm{n}=6$ for each group; $P<.001$, intimal thickness of E2F decoy ODN versus vehicle or scrambled ODN groups at 6 weeks $[*]$ and 6 months $[\#]$ ).

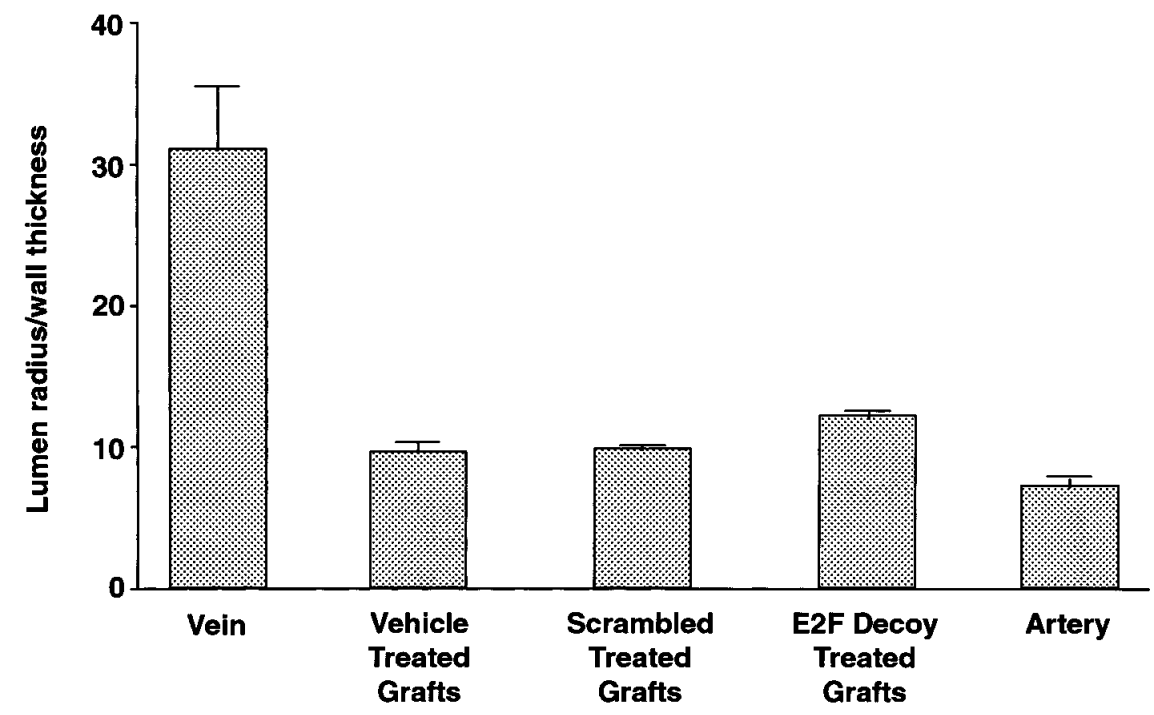

Fig 4. Tangential wall stress in rabbit carotid artery and vein grafts 6 months after grafting in cholesterol-fed rabbits, as reflected by the ratio of lumenal radius to wall thickness. No statistically significant difference was found between E2F decoy ODN-treated grafts when compared with vehicle or scrambled ODN-treated grafts $(n=6$ for each group).

earlier time points persisted even at 6 months in grafts that had undergone cell-cycle gene blockade with a single E2F decoy ODN transfection at the time of the operation (Fig 3).

The aggressive development of atherosclerotic lesions has been well characterized in rabbit vein grafts that undergo neointimal hyperplasia in the presence of hypercholesterolemia, whereas plaque formation and foam cell lesions are not observed, even in long-term grafts placed in normal rabbits. ${ }^{14,15}$ Vein graft wall remodeling stabilizes between 4 and 12 weeks after implantation, as rates of VSMC proliferation return to 


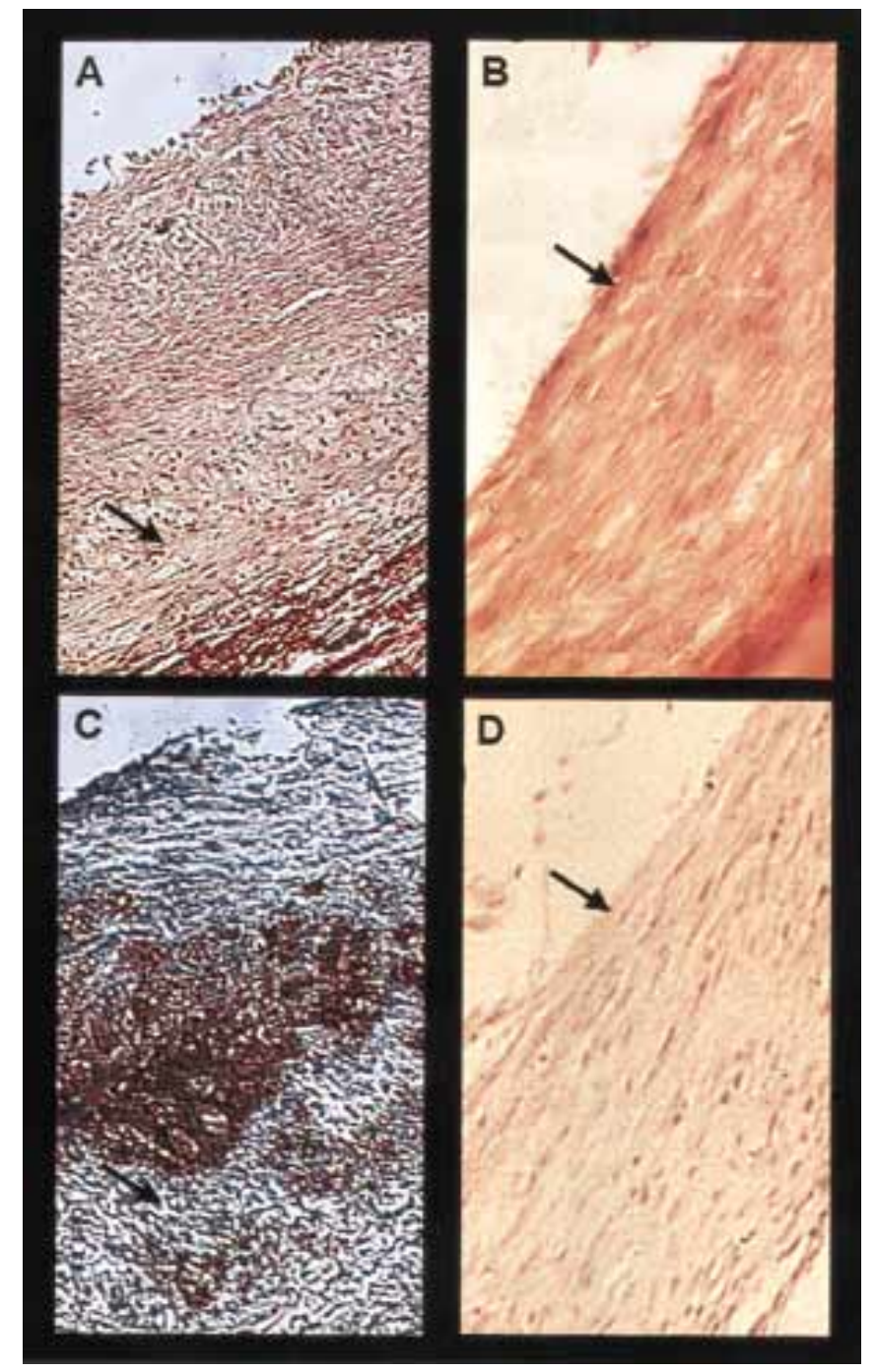

Fig 5. Light micrographs of scrambled ODN-treated (A and C) and E2F decoy ODN-treated (B and D) vein grafts 6 months after grafting in cholesterol-fed rabbits. Oil red-O staining demonstrates a pronounced, lipid-rich, foam cell lesion covered by a cap of fibrous tissue in scrambled ODN-treated grafts (C). E2F decoy ODN-treated grafts remained free of macroscopic plaque and foam cell lesions. Arrows indicate locations of internal elastic laminae (hematoxylin-van Geison stain: A and B, original magnification 400×).

low levels and neointimal thickening ceases. We postulated, however, that an increased susceptibility to accelerated atherogenesis persists in the hemodynamically stabilized graft wall that has undergone neointimal hyperplasia and in which abnormal VSMC and endothelial cell phenotypes persist and that the alternative form of graft remodeling achieved after E2F decoy ODN treatment does not involve this development of long-term proatherogenic state. Normocholesterolemic rabbits that had undergone interposition grafting with vehicle-treated, scrambled ODN-treated, and E2F decoy ODN-treated veins were then administered a $1 \%$ cholesterol diet for 6 weeks, starting 6 months after the operation. At the time of harvest, grafts in both control groups exhibited atherosclerotic lesion formation indistinguishable from 6-week grafts placed in hypercholesterolemic rabbits, whereas the gross and microscopic appearance of E2F decoy ODN-treated grafts reflected a persistent resistance to this accelerated disease process (Fig 6). Furthermore, the introduction of elevated cholesterol levels did not induce new neointimal hyperplasia in E2F decoy ODN-treated grafts $(10 \pm 3$ $\mu \mathrm{m})$ that had achieved mechanical stabilization through medial hypertrophy when compared with vehicle-treat- 


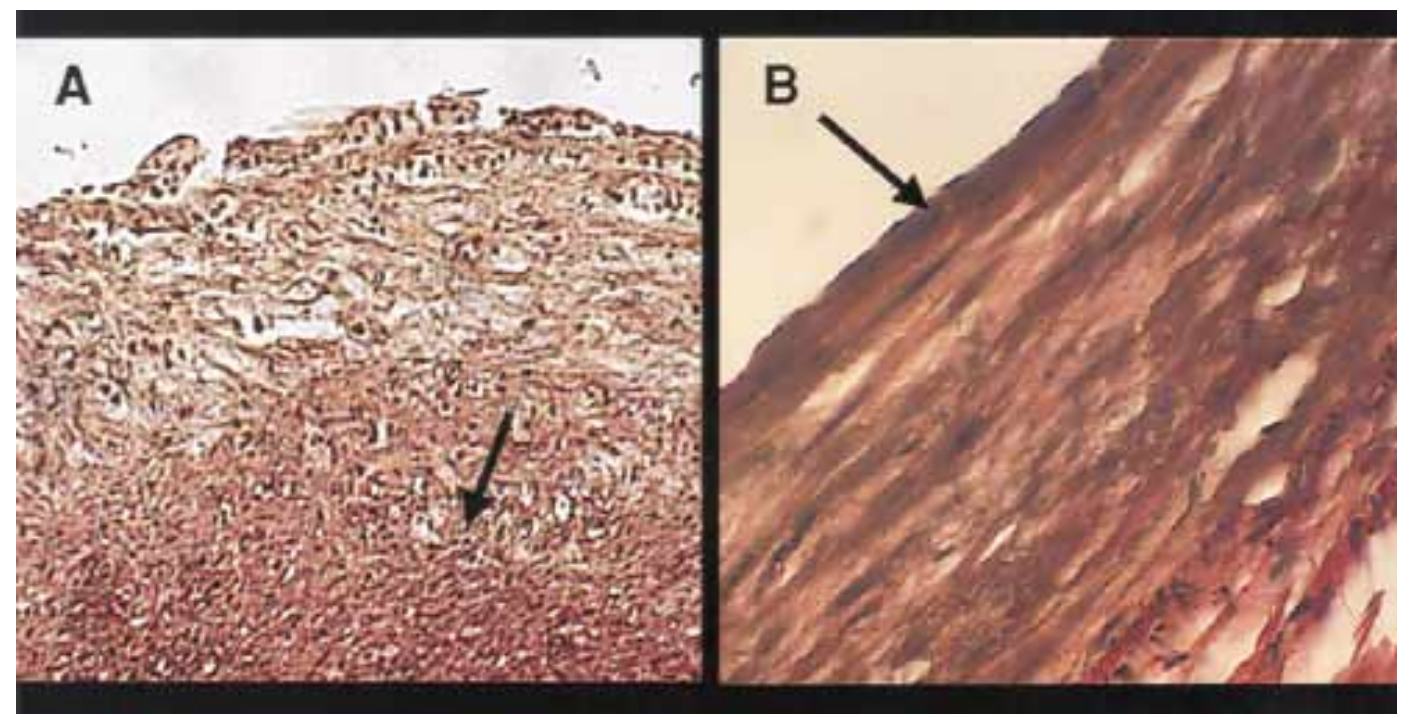

Fig 6. Light micrographs of scrambled ODN-treated (A) and E2F decoy ODN-treated (B) vein grafts in normocholesterolemic rabbits rendered hypercholesterolemic 6 months after grafting. A pronounced fibrous cap is seen in the scrambled ODN-treated graft, whereas the E2F decoy ODN-treated graft remains free of macroscopic plaque. Arrows indicate locations of internal elastic laminae (hematoxylin-van Geison stain: original magnification $400 \times)$.

ed grafts $(138 \pm 12 \mu \mathrm{m})$ and scrambled ODN-treated grafts $(130 \pm 14 \mu \mathrm{m})$.

\section{Discussion}

In this study we have demonstrated that transfection of vein grafts with a single ODN designed to block the activity of the critical cell-cycle regulatory transcription factor E2F-1 can achieve therapeutic graft wall adaptation without a need for multiple antisense ODNs against individual cell-cycle genes. In addition, our results indicate that the effect of this alternative approach to cellcycle gene inhibition modulates not only patterns of cell division after operation but phenotype as well because E2F decoy ODN-treated grafts resisted not only the development of neointimal hyperplasia but also dietinduced atherosclerosis. Furthermore, this vein graft engineering strategy was implemented by using an ex vivo DNA delivery approach, without exogenous lipid formulations, viral protein components, live viral preparations, or other foreign materials that have been used in previous attempts at modifying vein graft pathogenesis $^{5,6,16,17}$ and therefore represents a particularly safe means of potentially applying our growing knowledge of vascular molecular biology to the treatment of human disease.

Although accelerated in comparison with native arterial atherosclerosis, occlusive vein graft disease is a progressive process that manifests over the first months to years after grafting. Although a single delivery of
ODN cannot be expected to remain available to influence gene expression over this prolonged time course, we have hypothesized that a pattern of adaptive vascular remodeling is set during the days after vein grafting that will lead the vessel either toward or away from the accelerated disease process. Many forms of vascular injury have been associated with VSMC activation that leads to neointima formation. The phenotypic activation of vascular cells in response to the injuries associated with surgical trauma, ischemia, initial overdistention, and perioperative inflammation involves such proatherosclerotic developments as adhesion molecule upregulation, cytokine secretion, heightened oxidative stress, and shifts in endothelium-dependent vasoreactivity. ${ }^{18-21}$ The neointima, with its phenotypically activated VSMCs and the leukocytes subsequently attracted by its proinflammatory environment, yields a substrate for the accelerated atherogenesis seen in human and experimental vein grafts. In contrast to the neointimal thickening stimulated by the vascular injuries associated with vein grafting, arterial models of hypertension have revealed a mechanism of medial hypertrophy that allows adaptation to chronically increased hemodynamic stress without neointimal layer formation..$^{22,23}$ This medial hypertrophy involves both increased protein synthesis and proliferation of VSMCs in the medial layer that, in the absence of acute vascular injury, do not take on an activated phenotype and do not migrate to form a neointimal lesion. 
We have recently observed that the susceptibility of VSMCs to cytokine-induced activation is significantly reduced after early cell-cycle arrest. ${ }^{24}$ By dampening this initial VSMC activation in response to acute injuries of grafting, inhibition of cell-cycle regulatory gene expression has allowed grafts to respond to the more chronic hemodynamic stress of the arterial circulation through medial thickening that does not involve the expression of proinflammatory genes. The eventual dissipation of E2F decoy activity allows proliferation of medial VSMCs to contribute to this process in the absence of acute vascular injuries that can trigger VSMC activation, migration, and neointima formation. In the current study vein grafts that had received a single intraoperative transfection with E2F decoy ODNs and that initiated an adaptive response that did not involve neointimal hyperplasia continued to resist neointima formation and atherosclerosis as long as 6 months after operation. These results indicate that once hemodynamic stability has been achieved, the vein graft wall is no longer subject to stimuli for vascular cell activation and neointimal hyperplasia, and that long-term resistance to neointimal disease and atherosclerosis may therefore be observed in a clinical setting.

The cholesterol-fed rabbit offers a model of vein graft atherosclerosis that produces lesions reminiscent of those seen in human disease. ${ }^{15}$ In addition, as in human vein grafts, the time course of this lesion formation is greatly accelerated compared with native arterial disease because plaque forms in hypercholesterolemic rabbit grafts over the course of weeks in contrast to the months required for atherosclerosis in the rabbit aorta. ${ }^{15,25}$ This experimental atherogenesis, however, begins during the early postoperative period in the context of active vascular cell stimulation and proliferative activity at levels higher than those present during the course of human graft atherosclerotic lesion development. We therefore examined whether this increased susceptibility to atherogenesis was sustained as a chronic characteristic of the graft neointima and whether reduction of the neointimal component of vein graft adaptation could therefore be expected to influence the grafts' long-term likelihood of disease. Rabbit vein grafts were found to develop atherosclerotic lesions on exposure to hypercholesterolemia, even after a 6-month period of stabilization in the arterial circulation. More important, the avoidance of neointima formation and the alternative adaptation undertaken by grafts that had been treated once with E2F decoy ODNs at the time of operation 6 months earlier prevented lesion formation, even after a delayed exposure to a proatherogenic environment.
In summary, we have demonstrated that the longterm biology of the vessel wall is dramatically influenced by the events of the vein graft harvest and implantation into the arterial circulation and that a long-term protective shift from a pathologic phenotype can be achieved by a strategy of transient but critical alteration of specific vascular cell genetic activity around the time of operation. Vein graft disease offers a somewhat unique opportunity for translation of a molecular therapeutic strategy to human application. Unlike native arterial atherosclerosis, vein graft disease is known to begin at a discrete point in time, a time at which the tissue is readily accessible to direct intervention and therefore to possible manipulation of the genetic machinery driving pathogenesis. In addition, vein graft disease results from exposure of relatively normal tissue to an abnormal physiologic environment and is therefore more amenable to accurate experimental modeling than the more complex and multifactorial human atherosclerosis in native arteries. Furthermore, the ex vivo availability of the vein grafts allows easier application in the human clinical setting of DNA transfer techniques, such as pressure-mediated delivery, that provide highly efficient, targeted, and safe genetic manipulation. The observations reported in this study, coupled with freedom from viral and other potentially hazardous DNA transfer methodologies, have provided a foundation for the initiation of clinical studies of this gene-based approach for the amelioration of human vein graft disease. ${ }^{26}$ Future large-scale studies may provide further insight into the feasibility of intraoperative manipulation of the genetic roots of a common human cardiovascular disease.

Received for publication March 15, 2000; revisions requested June 26, 2000; revisions received Aug 23, 2000; accepted for publication Aug 31, 2000.

Address for reprints: Victor J. Dzau, MD, Tower 1, Office of the Chairman, Department of Medicine, Brigham and Women's Hospital/Harvard Medical School, 75 Francis St, Boston, MA 02115 (E-mail: vdzau@ rics.bwh.harvard.edu).

\section{REFERENCES}

1. Campeau L, Enjalbert M, Lesperance J, Bourassa MG, Kwiterovich P Jr, Wacholder S, et al. The relation of risk factors to the development of atherosclerosis in saphenous vein bypass grafts and the progression of disease in the native circulation: a study 10 years after aortocoronary bypass surgery. $\mathrm{N}$ Engl J Med 1984;311:1329-34.

2. Grondin CM. Late results of coronary artery grafting: is there a flag on the field? J Thorac Cardiovasc Surg 1984;87:161-6.

3. Reardon MJ, Conklin LD, Reardon PR, Baldwin JC. Coronary artery bypass conduits: review of current status. J Cardiovasc Surg 1997;97:916-31. 
4. Clowes AW, Reidy MA. Prevention of stenosis after vascular reconstruction: pharmacologic control of intimal hyperplasia. J Vasc Surg 1991;13:885-90.

5. Fulton GJ, Davies MG, Koch WJ, Dalen H, Svendsen E, Hagan $\mathrm{P}-\mathrm{O}$. Antisense oligonucleotide to proto-oncogene c-myb inhibits the formation of intimal hyperplasia in experimental vein grafts. J Vasc Surg 1997;24:453-63.

6. Bai H, Morishita R, Kida I, Yamakawa T, Zhang W, Aoki M, et al. Inhibition of intimal hyperplasia after vein grafting by in vivo transfer of human senescent cell-derived inhibitor-1 gene. Gene Ther 1998;5:761-9.

7. Mann MJ, Gibbons GH, Kernoff RS, Diet FP, Tsao PS, Cooke JP, et al. Genetic engineering of vein grafts resistant to atherosclerosis. Proc Natl Acad Sci USA 1995;92:4502-6.

8. Chellappan SP, Hiebert S, Mudryj M, Horowitz JM, Nevins JR. The E2F transcription factor is a cellular target for the RB protein. Cell 1991;65:1053-61.

9. DeGregori J, Kowalik T, Nevins JR. Cellular targets for activation by the E2F1 transcription factor include DNA synthesis- and G1/S-regulatory genes. Mol Cell Biol 1995;15:4215-24.

10. Weinberg RA. The retinablastoma protein and cell cycle control. Cell 1995;81:323-30.

11. Nevins JR. Towards an understanding of the functional complexity of the E2F and retinoblastoma families. Cell Growth Differ 1998;9:585-93.

12. Morishita R, Gibbons GH, Horiuchi M, Ellison KE, Nakama $\mathrm{M}$, Zhang L, et al. A gene therapy strategy using a transcription factor decoy of the E2F binding site inhibits smooth muscle proliferation in vivo. Proc Natl Acad Sci USA 1995;92:5855-9.

13. Mann MJ, Gibbons GH, Hutchinson H, Poston RS, Hoyt EG, Robbins RC, et al. Pressure-mediated oligonucleotide transfection of rat and human cardiovascular tissues. Proc Natl Acad Sci USA 1999;96:6411-6.

14. Batellier J, Wassef M, Merval R, Duriez M, Tedgui A. Protection from atherosclerosis in vein grafts by a rigid external support. Arterioscler Thromb 1993;13:379-84.

15. Zwolak RM, Kirkman TR, Clowes AW. Atherosclerosis in rabbit vein grafts. Arteriosclerosis 1989;9:374-9.
16. George SJ, Baker AH, Angelini GD, Newby AC. Gene transfer of tissue inhibitor of metalloproteinase-2 inhibits metalloproteinase activity and neointima formation in human saphenous veins. Gene Ther 1998;5:1552-60.

17. Schwartz LB, Moawad J, Svensson EC, Tufts RL, Meyerson SL, Baunoch D, et al. Adenoviral-mediated gene transfer of a constitutively active form of the retinoblastoma gene product attenuates neointimal thickening in experimental vein grafts. J Vasc Surg 1999;29:874-81

18. Cox JL, Chaisson DA, Gotleib AI. Stranger in a strange land: the pathogeneis of saphenous vein graft stenosis. Prog Cardiovasc Dis 1991;34:45-68.

19. Kohler TR, Kirkman TR, Gordon D, Clowes AW. Mechanism of long-term degeneration of arterialized vein grafts. Am J Surg 1990;160:257-61.

20. Loppnow H, Libby P. Proliferating or interleukin 1-activated human vascular smooth muscle cells secrete copious interleukin 6. J Clin Invest 1990;85:731-8

21. Majesky MW, Giachelli CM, Reidy MA, Schwartz SM. Rat carotid neointimal smooth muscle cells reexpress a developmentally regulated mRNA phenotype during repair of arterial injury. Circ Res 1992;71:759-68.

22. Heagerty AM. Changes in vascular morphology in essential hypertension. J Hum Hypertens 1991;5(Suppl 1):3-8.

23. Koffi I, Safer ME, Labat C, Lacolley P, Benetos A, Mourad JJ. Arterial structural changes with verapamil in spontaneously hypertensive rats. Am J Hypertens 1999;12:732-8.

24. Braun-Dullaeus RC, Mann MJ, von der Leyen HE, Dzau VJ. Cell cycle dependent modulation of VCAM-1 expression in vascular smooth muscle cells is transcriptionally regulated through IRF-1 [abstract]. Circulation 1998;98(Suppl):I-601.

25. Cooke JP, Singer AH, Tsao P, Zera P, Rowan RA, Billingham ME. Antiatherogenic effects of $\mathrm{L}$-arginine in the hypercholesterolemic rabbit. J Clin Invest 1992;90:1168-72.

26. Mann MJ, Whittemore AD, Donaldson MC, Belkin M, Conte MS, Polak JF, et al. Ex-vivo gene therapy of human vascular bypass grafts with E2F decoy: the PREVENT single-centre, randomised, controlled trial. Lancet 1999;354:1493-8. 